\title{
Wavefront outer scale deduced from interferometric dispersed fringes
}

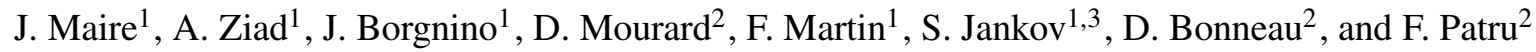 \\ ${ }^{1}$ Lab. Univ. d'Astrophysique de Nice (LUAN) UMR 6525 UNSA, Parc Valrose, 06108 Nice Cedex 2, France \\ e-mail: maire@unice.fr \\ 2 OCA, GEMINI, UMR6203, Avenue Nicolas Copernic, 06130 Grasse, France \\ 3 Astronomical Observatory, MSEP of Serbia 146007, Volgina 7, 11050 Belgrade, Serbia and Montenegro
}

Received 2 February 2005 / Accepted 4 November 2005

\section{ABSTRACT}

In addition to site characterization, measurements of critical atmospheric parameters are required to design and to optimize future adaptive optic systems and long-baseline interferometers. It is possible to estimate seeing conditions by processing data obtained with existing High Angular Resolution instruments. We report the results of joint observations with the GI2T interferometer and the GSM site-testing monitor performed over a period of several nights. We compared estimates of the wavefront outer scale done at various baselines as well as the seeing (Fried's parameter). We processed interferometric data by calculating power spectra of dispersed fringe images. Deduced measurements of the optical path difference lead to the estimates of the outer scale. We found that the outer scale values obtained from the GI2T data are mostly in the 5-30 m range, in good agreement with GSM measurements.

Key words. atmospheric effects - techniques: interferometric

\section{Introduction}

The fringes produced by a Michelson stellar ground-based interferometer are severely degraded by atmospheric turbulence. The characterization of wavefront fluctuations is very important for the design of the next generation of interferometers and large telescopes; this will enable the specification of basic parameters of these instruments and also the design of subsystems like adaptive optics, wavefront tip-tilt correctors and fringe trackers that overcome the limitations imposed by the atmosphere.

To estimate these incoming wavefront degradations, different instruments have been developped such as the DIMM (Differential Image Motion Monitor, Sarazin \& Roddier 1990) and the GSM (Generalized Seeing Monitor, Martin et al. 1994), which allow the monitoring of atmospheric optical parameters. As these facilities are not present in all observatories, it is possible to estimate seeing conditions by processing data obtained with existing High Angular Resolution (HAR) instruments like adaptive optic systems (Schöck et al. 2003; Fusco et al. 2004) or optical interferometers (see below). An initial calibration by simultaneous measurements with a seeing monitor is needed to overcome possible instrumental degradations.

A debate concerning the spatial coherence outer scale of the degraded wavefront $\mathcal{L}_{0}$ (called the outer scale hereafter) still exists (Avila et al. 1997). Its value is particularly important for modeling the Optical Path Difference (OPD) between the arms of an interferometer. In conditions of astronomical observations, experimental measurements of the variance of the phase difference made at small baselines are in excellent agreement with Kolmogorov turbulence spectra (Roddier 1981). In the case of long-baseline interferometers and Extremely Large Telescopes, the validity of this model may become questionable. Extensions of the Kolmogorov model beyond the inertial range have to take into account the outer scale influence. The GSM instrument deduces $\mathcal{L}_{0}$ on metric baselines from angleof-arrival fluctuations as a parameter of the von Kàrmàn (VK) turbulence spectrum. However, the validity of the VK model has never been proven. A long-baseline interferometer can be used to improve the knowledge of wavefront decametric properties. The first interferometric estimates of $\mathcal{L}_{0}$ were deduced from the spatial behaviour of the wavefront by Mariotti \& di Benedetto (1984): their long-baseline measurements show values of the pathlength difference lower than Kolmogorov model expectations. They estimated an $8 \mathrm{~m}$ outer scale with the $\mathrm{I}^{\mathrm{T}} \mathrm{T}^{1}$ interferometer. Davis et al. (1995) found significant departure from a Kolmogorov model with the $\mathrm{SUSI}^{2}$ interferometer when baselengths are above 5-10 m. Fitting the VK model to their data, we found a $53 \mathrm{~m}$ outer scale.

\footnotetext{
1 Interféromètre à 2 Télescopes.

2 Sydney University Stellar Interferometer.
} 
An other procedure in which the temporal power spectrum of fringe position is considered allowed Colavita et al. (1987) to estimate, with the Mark III interferometer, the value of the outer scale to be over two kilometers. Later, evidence for a decametric outer scale was presented by Buscher et al. (1995) with the same instrument. Nightingale \& Buscher (1991) obtained interferometric measurements of a metric outer scale at La Palma Observatory while Haniff et al. (1994) have found very large values at the same observatory. With the $\mathrm{ISI}^{3}$ interferometer, Bester et al. (1992) measured the outer scale in the range 5-20 m. Linfield et al. (2001) found most values in the 10-25 m range with the PTI ${ }^{4}$ interferometer.

Other methods have been used to estimate $\mathcal{L}_{0}$ : using the GI2T $^{5}$ interferometer, Berio et al. (1997) measured the visibility losses due to the spectral decorrelation; Ziad et al. (2004) derived $\mathcal{L}_{0}$ values from fringe speed measurements of the PTI interferometer and compared them with simultaneous GSM measurements. Except for this last work, no independent outer scale monitor was run simultaneously during these measurements. The use of different instruments limit misinterpretations, and give simultaneous spatial and temporal information on various scales. The joint observation and comparison of such data could check the validity of the VK model by measurement of the phase structure function.

Dispersed fringe images are obtained using the GI2T/Regain ${ }^{6}$ and VLTI $^{7} /$ Amber $^{8}$ interferometers. The GI2T interferometer can be used to measure the phase structure function at several baselines assuming the stationarity of turbulence. With the VLTI/Amber instrument, it will soon be possible to measure it simultaneously at 3 different baselines up to $200 \mathrm{~m}$.

Here, we processed the GI2T data to monitor the spatial coherence parameters of the wavefront, $r_{0}$ and $\mathcal{L}_{0}$. The method used can be applied for any dispersed fringe data as shown in Sect. 2. We briefly describe the instrument and we examine errors that can affect our estimates in Sect. 3. GI2T results are compared to those obtained with the GSM instrument in Sect. 4. A discussion and perspectives are given in Sect. 5.

\section{Method of $\mathcal{L}_{0}$ estimation from interferometric data of dispersed fringes}

In the context of optical astronomy, the theoretical phase structure function $D_{\phi}$ describes the wavefront distortions due to the light propagation path. This function is defined as the meansquared difference of the phase fluctuations $\phi$ observed at two points separated by $\vec{B}$,

$D_{\phi}(\vec{B})=\left\langle[\phi(\vec{r})-\phi(\vec{r}+\vec{B})]^{2}\right\rangle$

where the brackets \langle\rangle denote an ensemble average.

\footnotetext{
${ }^{3}$ Infrared Spatial Interferometer.

${ }^{4}$ Palomar Testbed Interferometer.

5 Grand Interféromètre à 2 Télescopes.

${ }^{6}$ Recombinateur du Grand Interféromètre.

7 Very Large Telescope Interferometer.

8 Astronomical Multi-Beam Recombiner.
}

If the phase fluctuations are averaged over two circular apertures of diameter $D$, this function can be deduced from the phase power spectrum $W_{\phi}(\vec{u})$ (Conan et al. 2000), and becomes

$$
\begin{aligned}
D_{\phi}(\vec{B})=4 \pi \int_{0}^{+\infty} u W_{\phi}(\vec{u})[1 & \left.-J_{0}(2 \pi B u)\right] \\
& \times\left[\frac{2 J_{1}(\pi \mathrm{D} u)}{\pi \mathrm{D} u}\right]^{2} \mathrm{~d} u
\end{aligned}
$$

where $u$ is the modulus of the spatial frequency $\vec{u}$. $J_{0}$ and $J_{1}$ represent respectively the zeroth and first order Bessel functions of the first kind.

Atmospheric OPD fluctuations between the light beams coming from the arms of an interferometer depend on the turbulence parameters (Roddier 1981) as

$\sigma_{\mathrm{OPD}}=\frac{\lambda}{2 \pi} \sqrt{D_{\phi}(\vec{B})}$

The value of $\sigma_{\mathrm{OPD}}$ is needed for the design and the optimization of fringe trackers. For large baselines, the VK model strongly reduces the value of $\sigma_{\mathrm{OPD}}$ that can be deduced in the frame of the Kolmogorov model. A relation between $\sigma_{\mathrm{OPD}}$ obtained with the two models is developed in Appendix A.

In the case of an interferometer with two circular apertures of diameter $D$, large enough to have $D \gg r_{0}$ and sufficiently distant by a baseline $B$, the expression of the image power spectrum is given by (Roddier \& Lena 1984; Mourard et al. 1994)

$$
\begin{aligned}
\left\langle|\overrightarrow{I(\vec{f})}|^{2}\right\rangle=\exp ( & \left.-D_{\phi}(\vec{f})\right)+\frac{\sigma_{\mathrm{c}}}{2 s}\left[T_{0}(\vec{f})\right. \\
& \left.+\frac{V^{2}}{2}\left(T_{0}\left(\vec{f}+\overrightarrow{f_{0}}\right)+T_{0}\left(\vec{f}-\overrightarrow{f_{0}}\right)\right)\right]
\end{aligned}
$$

where $\overrightarrow{f_{0}}=\frac{\vec{B}}{\lambda}$ is the angular frequency related to the baseline, $s=\iint T_{0}(\vec{f}) \mathrm{d}^{2} \vec{f}$ is the sub-pupil area, and $T_{0}(\vec{f})$ is the Modulation Transfer Function (MTF) of a sub-pupil, $\sigma_{\mathrm{c}}(\lambda)=\iint \exp \left(-D_{\phi}(\vec{f})\right) \mathrm{d}^{2} \vec{f}$ and $V$ is the fringe visibility amplitude. This analytical expression of the image power spectrum is obtained assuming that the wavefront complex amplitude is a Gaussian process. In the case of atmospheric fluctuations, the log-normal model is in better agreement with experimental results than the normal model (Aime et al. 1979), but does not lead to analytical solutions and requires numerical computations.

The classical representation of the image power spectrum (Fig. 1, Mourard et al. 1994) shows the distinct parts expected in the case of images dispersed by a spectograph. A low frequency part called the "seeing peak", centred on 0 , depends only upon the atmospheric conditions. A second low frequency part called the "speckle peak", centred on 0 , depends on the atmospheric conditions and upon the shape of the pupils; its cut-off frequency $D / \lambda$, is due to properties of image formation. When fringes are present in the images, the power spectrum has two high frequency peaks, centred at $\pm B / \lambda$ with an extent of $\pm D / \lambda$. They depend upon the turbulence (proportionally to $\left.\sigma_{\mathrm{c}}(\lambda)\right)$ and upon the shape of the pupils (Eq. (4)). 


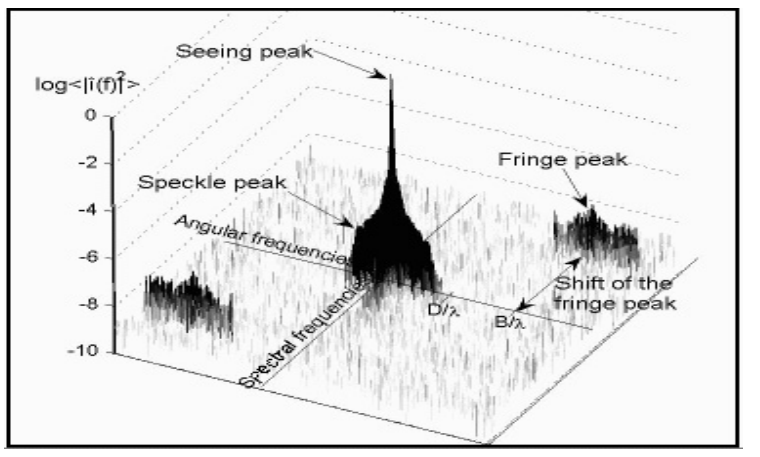

Fig. 1. Example of a real power spectrum averaged over short exposure dispersed images for the GI2T interferometer with two $1.5 \mathrm{~m}$ apertures measured at $00 \mathrm{~h} 41^{\prime}(\mathrm{UT})$ in the night of June 7, 2003. Observation of $\alpha$ Lyr.

High spectral resolution is obtained by spectral dispersion of the fringes. The fringe parallel to the direction of dispersion corresponds to the zero path difference. As the distance that separates two adjacent fringes rises with the wavelength, the tilt of a fringe in a narrow spectral bandwidth is a measure of the instantaneous OPD as described by Koechlin et al. (1996). The GI2T fringe tracker detects the corresponding shift of the fringe peak in the power spectrum (Fig. 1) to deduce the OPD between the two arms of the interferometer.

This global OPD includes contributions of instrumental and atmospherical effects. It is neccessary to remove instrumental effects by filtering out their contribution. Introducing the phase power spectrum of the VK model (Eq. (A.4)) in Eq. (3) and using a $r_{0}$ measurement, one can deduce an estimation of the outer scale. This method is appropriate given that the standard deviation of the OPD strictly increases with $\mathcal{L}_{0}$ for a fixed baseline.

The wavefront coherence diameter $r_{0}$ is deduced from the low frequency part of the experimental spectrum. Using the Kolmogorov model of turbulence, the expression of $\sigma_{\mathrm{c}}(\lambda)$ depends upon $r_{0}$ (Roddier 1981) as

$\sigma_{\mathrm{c}}(\lambda)=0.342\left(\frac{r_{0}}{\lambda}\right)^{2}$

and leads to the $r_{0}$ estimation by integrating the seeing peak. At frequencies lower than $r_{0} / \lambda$, both seeing and speckle peaks contribute to the spectrum. It follows that this method requires the reconstruction of the low frequency part of the speckle peak by using an extrapolation of a higher frequency experimental part, for example over the interval $\left[\frac{D}{2 \lambda}, \frac{D}{\lambda}\right]$. This reconstruction is better when $D \gg r_{0}$. The reliability of this reconstruction depends on the quality and the number of images used for the calculation of the power spectrum as well as the actual geometry of the pupils.

This $r_{0}$ estimation does not take into account the outer scale influence. It is based on Fried's definition and $r_{0}$ characterizes the optical resolution imposed by the atmosphere and the telescope in the framework of the Kolmogorov model.

\section{Experimental data}

The observing campaign regrouping the GI2T interferometer and the GSM monitor was performed during several nights at the Calern Observatory in the south of France (altitude $1170 \mathrm{~m}$, latitude $43^{\circ} 44^{\prime} 55^{\prime \prime} \mathrm{N}$, longitude $\left.06^{\circ} 55^{\prime} 30^{\prime \prime} \mathrm{E}\right)$. The two instruments are described below. The steps performed to extract wavefront parameters from interferometric measurements are reported in the second subsection. We estimate errors that affect the outer scale and seeing measurements with the GI2T data.

\subsection{Instruments}

\subsubsection{The GI2T/REGAIN interferometer}

The GI2T interferometer, part of the "Observatoire de la Côte d'Azur", has been detailed elsewhere (Mourard et al. 2001), the most important aspects related to seeing measurement are repeated here. The two telescopes have a diameter of $1.52 \mathrm{~m}$ and a $0.2 \mathrm{~m}$ central obscuration. Light is collected from a single star and is sent towards a central laboratory where the beams are combined. The baseline is oriented to the North-South and can vary continuously from $12 \mathrm{~m}$ to $65 \mathrm{~m}$. It is equipped with a spectrograph to achieve simultaneous high spatial and spectral resolutions ( $\mathrm{X}-\lambda$ mode configuration). Light is received in two spectral channels constituted by two photon-counting detectors with a $764 \times 574$ pixel array.

A multichromatric mode or a dispersed fringe mode can be used for observations. In order to increase sensitivity by using the whole speckle pattern, the multichromatric mode records filtered images in discrete adjacent spectral bands. To increase the spectral resolution the dispersed fringe set includes a one speckle wide slit in the recombined image plane. This allows three different spectral resolutions $R=30000,5000$ and 1500 . This mode was used for measurements of the wavefront optical parameter simultaneously with the GSM instrument.

The REGAIN entrance optical table contains a delay line for optical path compensation. For our observations, the correction in real-time of the path difference was not activated but the delay line followed the diurnal movement which depends on the coordinates of the star, the universal time and the position of the telescopes. The entrance optical table contains a rotator for field rotation and atmospheric dispersion compensations. The beam combiner carries out a Michelson pupil reconfiguration.

\subsubsection{The GSM instrument}

The GSM instrument allows the evaluation of the optical parameters of the perturbed wavefront by measuring Angleof-Arrival (AA) fluctuations. Four telescopes allow AA measurements at different points of the wavefront with baselines of $0.25 \mathrm{~m}$ to $2.1 \mathrm{~m}$. After computing AA spatio-temporal correlations, it estimates the seeing $\epsilon_{0}$, the outer scale $\mathcal{L}_{0}$, the isoplanetic angle $\theta_{0}$ and the coherence time $\tau_{0_{\mathrm{AA}}}$ (Ziad et al. 2004).

This instrument consists of four identical modules, equipped with $0.1 \mathrm{~m}$ telescopes installed on equatorial mounts. Telescopes point at the same star and AA fluctuations are measured by means of flux modulation that is produced by the 
displacement of the star image over a Ronchi grating. Detected photons are counted by a photomultiplier working in photon counting mode, allowing fast and continuous monitoring of AA fluctuations. Two modules share the same mount which avoids instrumental errors like vibrations due to wind effect on modules or telescope tracking. The AA fluctuations are measured with a $5 \mathrm{~ms}$ exposure time during an acquisition time of two minutes. A correction of this exposure time on the $r_{0}$ and $\mathcal{L}_{0}$ estimates is performed (Ziad et al. 2000).

The Fried parameter $r_{0}$ is measured from the AA differential variances obtained with modules sharing the same mount.

The outer scale $\mathcal{L}_{0}$ is determined for each pair of modules (6 different baselines) by computing the ratio of AA covariance to the differential AA variance. These normalized covariances are then compared to the grid of theoretical covariances calculated with the VK model for different baseline coordinates and also for different values of $\mathcal{L}_{0}$. The adopted $\mathcal{L}_{0}$ value is the median of the 6 obtained values. A detailed description of the GSM experiment is given by Ziad et al. (2000).

\subsection{Data processing}

Methods used in this paper to extract the wavefront parameters from dispersed fringes are based on the calculation of the power spectrum of the short exposure images obtained from the GI2T interferometer. The $\mathrm{X}-\lambda$ mode configuration permits one to obtain both high spatial and spectral resolution. Each image corresponds to a $20 \mathrm{~ms}$ exposure time, and is processed in realtime to generate a stream of 2-dimensional photon coordinates. Each image has a size determined by the slit height and the spectral bandwidth. The result is that the power spectrum has a set of angular and spectral frequency axes (see Fig. 2). The data files correspond to sequences of about $3 \mathrm{mn}$, this duration being defined by the telescope driving system.

We first calculate the sum of the 2-D autocorrelation of the dispersed fringe images (Mourard et al. 1994). The WienerKhinchin theorem shows that this autocorrelation and the power spectrum of the images are Fourier transform pairs; then, the power spectrum is determined by applying a 2-D Fourier transform to this sum. This method allows a very fast calculation of the power spectrum in comparison to the calculation of the Fourier transform of each image. Indeed, the algorithm for the computation of the image autocorrelation takes into account that the number of photons is small in comparison with the number of pixels per image. The steps of this process are represented in Fig. 2.

For $r_{0}$ estimation, the whole file $(\approx 9000$ images) is processed in order to compute the power spectrum. During an observation, images of sub-pupils are also recorded in order to determine the MTF $T_{0}(\vec{f})$ of a sub-pupil (Eq. (4)). For the separation of the seeing peak from the speckle peak, a least-square method is performed between the power spectrum values and the sub-pupil MTF over the frequencies $\left[\frac{D}{2 \lambda}, \frac{D}{\lambda}\right]$. It allows the calculation of the speckle peak over the frequencies $\left[0, \frac{D}{2 \lambda}\right]$. The seeing peak is determined by subtracting the speckle peak obtained from the power spectrum (Mourard et al. 1994).

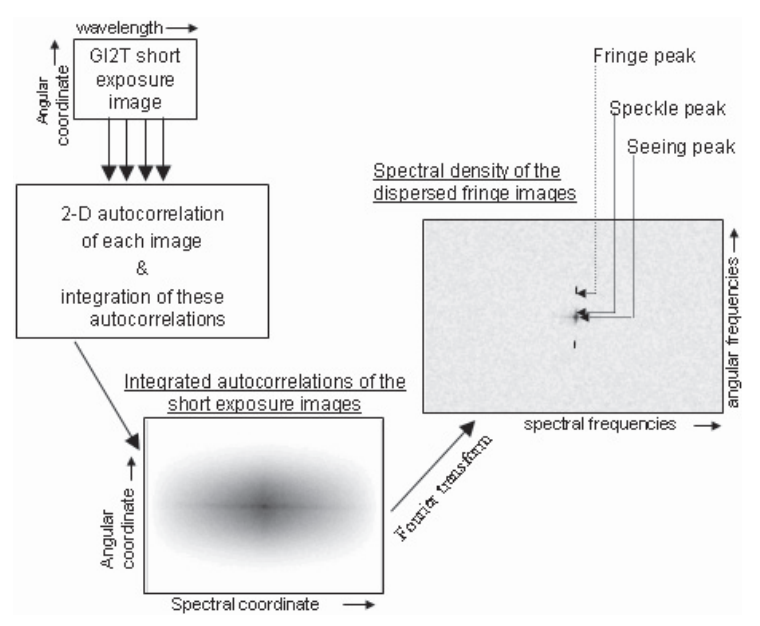

Fig. 2. Calculation of the power spectrum of the short exposure images.

Each data file leads to one estimation of $\mathcal{L}_{0}$. Indeed, for each sequence, we calculate about 180 continuous path difference values deduced from image power spectra, as described in Sect. 2, and performed by steps of $1 \mathrm{~s}$ and for an integration time of $8 \mathrm{~s}$. The dynamic range of the OPD measured is limited by the lowest temporal frequency reachable which depends on the number of images per sequence of acquisition; it was typically $5 \mathrm{mHz}$ in our run. The highest frequency $(0.5 \mathrm{~Hz})$ is determined by the time calculation step used in the process. Typically, a Kolmogorov model can be expected beyond this frequency (Buscher et al. 1995) in the temporal power spectrum of the fringe position.

\subsection{Error estimation}

Different errors can affect outer scale and seeing measurements deduced from the GI2T data.

The photometry of the images is degraded by the photon centroiding electronics of the camera that computes the coordinates of the photo-events: indeed, when several simultaneous photo-events are very close in the image, the photon centroiding device is unable to identify them properly and does not take into account all events. The photon centroiding hole appears at the center of the mean autocorrelation of images obtained with the camera. This hole becomes larger when the photon flux increases. This centroiding hole is removed in the autocorrelation by fitting a 2D polynomial function (Berio et al. 1998).

The photon noise bias is also corrected. Due to the short exposure time, the photo-events (a few hundred per image) are assumed to be independent and follow Poisson statistics. Consequently, it can be shown (Aime 2001) that the detected autocorrelation origin of the image takes into account a supplementary term, which is inversely proportional to the mean photo-events per image $N$. The power spectrum obtained is biased by a additional term $1 / N$ for all frequencies. We change the autocorrelation center value to correct it.

In order to automatically remove the power spectra that have fringes highly attenuated by atmospheric effects, or 
possible instrumental and star tracking problems, we calculated the signal-to-noise ratio (SNR) defined as the ratio of the mean value over the fringe peak extent to the noise level. This noise level is determined by averaging a part of the power spectrum without interferometric signals. Due to the spectral banwidth used during our observation, the sampling resolution of the spectral frequency axis corresponds to an OPD of $5 \mu \mathrm{m}$ per pixel. The maximum of the integration over the high spatial and spectral frequencies determines the position of the fringe peak in the power spectrum. The position of the center is calculated by a centroid algorithm using only the data within a "small" window around that peak. By processing 50 one-dimensional profiles of the fringe peak extent, this algorithm is able to determine the OPD with an accuracy of $1 / 20$ th of a pixel or $0.25 \mu \mathrm{m} \mathrm{rms}$. This OPD accuracy was found by applying the process of centroid determination on a simulated fringe peak that has the minimum SNR allowed for the detection. A typical value of $\sigma_{\mathrm{OPD}}$ in the GI2T case (Appendix A) gives a relative uncertainty of $8 \%$ on $\sigma_{\mathrm{OPD}}$ measurements.

\subsubsection{Error on $\mathcal{L}_{0}$ estimates}

The relative uncertainty on $\mathcal{L}_{0}$ can be deduced with the asymptotic solutions for long-baseline regimes of the phase structure function (Eq. (A.5)): when $D \ll \mathcal{L}_{0} / \pi \ll B$ the relative uncertainty on $\mathcal{L}_{0}$ is:

$\frac{\Delta \mathcal{L}_{0}}{\mathcal{L}_{0}}=\frac{6}{5} \frac{\Delta \sigma_{\mathrm{OPD}}}{\sigma_{\mathrm{OPD}}}+\frac{\Delta r_{0}}{r_{0}}$

Eq. (6) is appropriate in the case of the GI2T or interferometers with small apertures. When $\mathcal{L}_{0} / \pi \ll D \ll B$, it becomes

$\frac{\Delta \mathcal{L}_{0}}{\mathcal{L}_{0}}=\frac{6}{11} \frac{\Delta \sigma_{\mathrm{OPD}}}{\sigma_{\mathrm{OPD}}}+\frac{5}{11} \frac{\Delta r_{0}}{r_{0}}$

Eq. (7) is appropriate in the case of the VLTI or interferometers with large apertures when the value of the outer scale is small.

The limit of the method appears for small baselines, when $\mathcal{L}_{0} / \pi \ll D \ll B$ which is the least favorable case in the calculation of error bars. It makes it necessary to use long baselines to apply the method properly.

With two telescopes of an interferometer observing a distant source, the OPD measured is the sum of the atmospheric path difference and the internal path difference. Different sources of internal OPD can be considered, such as mechanical instabilities, star tracking errors or horizontal propagation in the arms of the interferometer.

Different characteristic times of the wavefront qualify the evolution of structures in the images. In the case of a single turbulent layer with wind velocity $\vec{v}$, the speckle life-time is (Roddier 1981)

$\tau_{0} \approx 0.31 r_{0} / v$.

The correlation time $T_{\mathrm{c}}$ of the larger structures of the wavefront is given by (Borgnino 2004)

$T_{\mathrm{c}} \approx \mathcal{L}_{0} / v$.

This correlation time is a relevant parameter for the temporal power spectrum of the fringe position: $F_{\mathrm{c}}=1 / T_{\mathrm{c}}$ is the knee

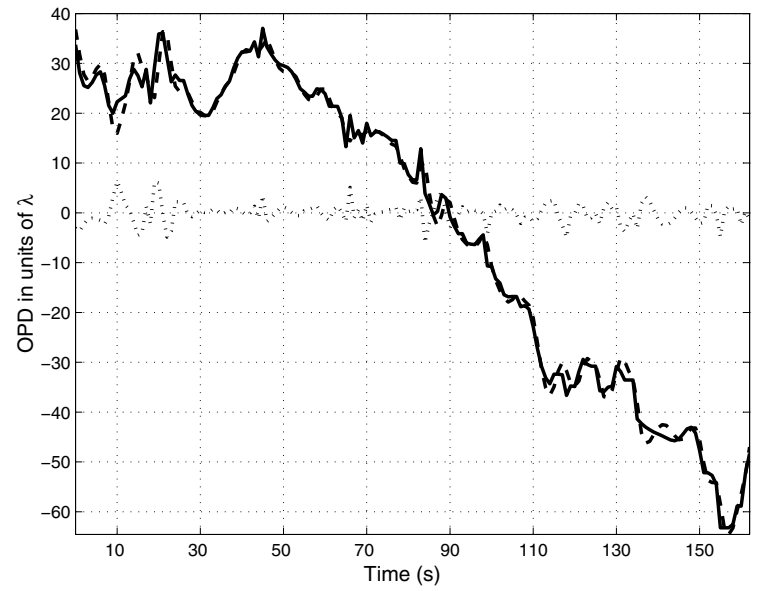

Fig. 3. Example of the high-pass filtering performed on the OPD to remove instrumental effects. The filtered OPD (dotted line) is obtained by subtracting the low frequency components (dashed line) from the total OPD measured (solid line). Observation of $\eta$ Uma the night of April 21, 2004 at 21h59' (UT).

frequency that limits the inertial range of turbulence. In the case of the VK model, this power spectrum saturates at frequencies lower than $F_{\mathrm{c}}$.

Measurements of vibrations of the GI2T delay line by accelerometers prior to the observation have shown that a very slow instrumental drift could be at the origin of additional OPD. The $3 \mathrm{~Hz}$ resonance frequency of the telescopes, which is not included in our temporal frequency range, has an amplitude sufficiently low to be considered negligible. Analysis of several OPD power spectra obtained during our run show that frequencies lower than $0.03 \mathrm{~Hz}$ have an asymptote noticeably steeper than the Kolmogorov model expectations. These low frequencies of OPD power spectra are affected by instrumental drifts and contain both atmospheric and instrumental contributions.

After a selection of fringe positions with good signal to noise ratio, a Chebyshev high-pass filtering (Parks \& Burrus 1987) of the pathlength difference discards the major part of the instrumental drift. Figure 3 shows a plot of a 160-s long record, and the OPD obtained by filtering out frequencies below $0.03 \mathrm{~Hz}$. The filtering cut-off frequency $f_{\mathrm{c}}$ must be higher than the highest instrumental contribution frequencies but lower than the characteristic frequency $F_{\mathrm{c}}$ of the fringe motion spectrum: the mean value of this latter frequency, calculated with GSM data (Eqs. (8) and (9)), is $F_{\mathrm{c}}=0.2 \mathrm{~Hz}$ for these nights of observation. Therefore the dynamic range of the OPD measured is restricted and the filtered OPD obtained does not take into account atmospheric long-period variations. If the entire night's data are examined, this low frequency contribution appears not significant as remarked first by Davis et al. (1995). By filtering out OPD variations with a cut-off frequency $f_{c}$ determined with $10 \%$ precision, we found a mean relative uncertainty of $7.5 \%$ on the $\sigma_{\mathrm{OPD}}$ estimates.

Colavita et al. (1987) defined the interferometric coherence time $T_{0}$ as the time interval during which the rms differential 
phase between both beams does not exceed $1 \mathrm{rad}$. They found the relation

$T_{0} \approx 0.81 r_{0} / v$

It follows from Eq. (8) that $T_{0} \approx 2.61 \tau_{0}$. The $\tau_{0}$ value is considered to be close to the wavefront coherence time $\tau_{0_{\mathrm{AA}}}$ measured by GSM. During our observation, its mean value was $8 \mathrm{~ms}$ in the visible. Hence the mean value of $T_{0}$ was larger than the exposure time used during the GI2T run. Thus, we will consider negligeable the influence of the exposure time on the measurement of the OPD.

In order to have a sufficient SNR to detect fringes, the power spectrum used for the OPD estimation is a time average of individual power spectra of each image. In the case of GI2T, we found that the best compromise between the number of images per power spectrum and the SNR is to compute power spectra after each series of 400 images in steps of $50 \mathrm{im}$ ages; this process will smooth OPD variations. To correct this effect, we calculated $\sigma_{\mathrm{OPD}}$ for a series of acquisitions with a different number of images per power spectrum; we used 400, 300, 200, 150 and 100 images. An exponential extrapolation of the different $\sigma_{\mathrm{OPD}}$ obtained can be applied to obtain a mean correction factor, corresponding to $\sigma_{\mathrm{OPD}}$ estimated with $50 \mathrm{im}$ ages per power spectrum. This process allows us to obtain $\sigma_{\mathrm{OPD}}$ estimates each second less than the characteristic time $T_{\mathrm{c}}$ of the fringe agitation estimated from GSM data during our run. Assuming that OPD samples are independent and follow a Gaussian probability law, the statistical relative error in the calculation of $\sigma_{\mathrm{OPD}}$ is given by (Frieden 1983) $\frac{1}{2}\left(\frac{2}{N-1}\right)^{1 / 2}$ where $N$ is the number of OPD samples per estimation of $\mathcal{L}_{0}$. The expected error is approximatively $5 \%$ for $3 \mathrm{~min}$ of acquisition.

Any wavefront phase fluctuations $\phi(\vec{r})$ observed just before the beam combiner can be considered as the sum of uncorrelated phase fluctuations, $\phi_{\mathrm{v}}(\vec{r})$ due to the vertical atmospheric propagation and $\phi_{\mathrm{h}}(\vec{r})$ due to the horizontal propagation inside the arm of the interferometer. In the recombined image plane, the variance of the phase difference is

$D_{\phi, \mathrm{m}}(\vec{B}) \approx D_{\phi, \mathrm{v}}(\vec{B})+D_{\phi, \mathrm{h}}(\vec{B})$

Using Eq. (A.5(b)), we can qualitatively compare the two right terms of Eq. (11) by calculating the ratio

$\frac{D_{\phi, \mathrm{h}}(\vec{B})}{D_{\phi, \mathrm{v}}(\vec{B})}=\left(\frac{\mathcal{L}_{0, \mathrm{~h}}}{\mathcal{L}_{0}}\right)^{5 / 3}\left(\frac{r_{0, \mathrm{v}}}{r_{0, \mathrm{~h}}}\right)^{5 / 3}$

where $r_{0, \mathrm{v}}$ is the atmospheric coherence diameter and $r_{0, \mathrm{~h}}$ is the wavefront coherence diameter due to the horizontal propagation. The outer scale $\mathcal{L}_{0, \mathrm{~h}}$ value inside the arms is limited by their cross section size. If we assume that propagation inside the horizontal arms follows the same properties as light vertical propagation, we can compare $r_{0, v}$ deduced from atmospheric $C_{N}^{2}(h)$ profile (Eq. (A.2)) and $r_{0, \mathrm{~h}}$ deduced from propagation with a constant $C_{N}^{2}$ value measured at $3 \mathrm{~m}$ above the ground. In this latter case, we do not take into account the zenith distance $\gamma$ dependence in Eq. (A.2). In-situ measurements made by Martin et al. (2000) at Paranal gave a mean value $C_{N}^{2}(3 \mathrm{~m}) \approx 6 \times 10^{-15} \mathrm{~m}^{-2 / 3}$. For a $7 \mathrm{~m}$ horizontal propagation, it gives $r_{0, \mathrm{~h}}=53.8 \mathrm{~cm}$ in the visible and the ratio $\left(r_{0, \mathrm{v}} / r_{0, \mathrm{~h}}\right)^{5 / 3}$ obtained in the same campaign is $7 \%$. If $\mathcal{L}_{0, \mathrm{~h}} \approx 2.5 \mathrm{~m}$, then the ratio $D_{\phi, \mathrm{h}}(\vec{B}) / D_{\phi, \mathrm{v}}(\vec{B}) \approx 0.2 \%$. For a propagation corresponding to a $32 \mathrm{~m}$ baseline, the ratio increases to $0.4 \%$. The error on the $\mathcal{L}_{0}$ estimates, due to the horizontal propagation, can be considered negligeable with regard to the atmospheric propagation.

\subsubsection{Error on $r_{0}$ estimates}

Several errors can be considered in the $r_{0}$ estimation from the power spectrum.

The narrow spectral bandwidth used gives a negligible attenuation of the power spectrum (Borgnino et al. 1989) taking into account the typical ratio $D / r_{0} \approx 15$ for GI2T observations.

The exposure time per image, $\tau$, should be maximum, given the low photon flux and smaller than $\tau_{0}$ in order to obtain a perfectly frozen speckle pattern. Roddier \& Roddier (1975) described the attenuation of the spectral components for several exposure times. They have shown experimentally that exposure times up to $20 \mathrm{~ms}$ have a negligible effect on the power spectrum. This effect becomes pronounced for $\tau_{0}$ less than one millisecond. They found that the effect is greater with a small telescope. The seeing peak should not be affected given the GI2T camera exposure time $\tau=20 \mathrm{~ms}$ compared to the mean $\tau_{0_{\mathrm{AA}}}=8 \mathrm{~ms}$ in the visible measured by GSM during the acquisition. The value of $r_{0}$ obtained by our method could be punctually overestimated due to high wind velocities and bad seeing (Roddier \& Roddier 1975).

For a long-baseline interferometer, horizontal progation inside the arms can lead to an error in the $r_{0}$ determination. Assuming that the horizontal propagation follows the same properties as vertical atmospheric propagation, the expression of $\sigma_{\mathrm{c}}$ (Eq. (5)) is modified by considering Eq. (A.2) as $\sigma_{\mathrm{c}}(\lambda)=\frac{0.342}{\lambda^{2}}\left(\left(r_{0, \mathrm{v}}^{-5 / 3}+r_{0, \mathrm{~h}}^{-5 / 3}\right)^{-3 / 5}\right)^{2}$.

For the same values of $r_{0, \mathrm{v}}$ and $r_{0, \mathrm{~h}}$ used in Sect. 3.3.1, the $r_{0}$ estimation using this modified $\sigma_{\mathrm{c}}$ is a $4 \%$ underestimate for a propagation corresponding to a $14 \mathrm{~m}$ baseline. This ratio increases to $9 \%$ for a $32 \mathrm{~m}$ baseline.

\section{Results}

In order to validate the methods of estimation described previously, we apply them to GI2T data and compare their results with GSM simultaneous measurements performed on several nights. Due to inadequate observational conditions, only three of the nights, 7th June 2003, 21st April 2004 and 22nd April 2004, gave exploitable data.

The GI2T configuration during the simultaneous observations is reported in Table 1. Some of the stars that were chosen in the scheduling of observations are different between the two instruments. This is due to instrumental constraints but their angular separation stays relatively small and does not change the seeing condition much. The addition of a new camera "Algolb" in 2004 at the GI2T focus allowed the testing of the 
Table 1. Configuration of the GI2T interferometer during the simultaneous observations.

\begin{tabular}{cccccc}
\hline \hline & $\begin{array}{c}\text { Star } \\
\text { obser- } \\
\text { ved by } \\
\text { GI2T }\end{array}$ & $\begin{array}{c}\lambda \\
(\mathrm{nm})\end{array}$ & $\begin{array}{c}\text { Base- } \\
\text { line } \\
(\mathrm{m})\end{array}$ & $\begin{array}{c}\text { First } \\
\text { acqui- } \\
\text { sition } \\
(\mathrm{TU})\end{array}$ & $\begin{array}{c}\text { Star } \\
\text { obser- } \\
\text { ved by } \\
\text { GSM }\end{array}$ \\
\hline 7th June & & & & & \\
2003 & $\alpha$ Lyr & 656 & 12.4 & $00 \mathrm{~h} 41^{\prime}$ & $\gamma$ Cyg \\
\hline 21 st April & $\beta \mathrm{Uma}$ & 529 & 12 & $20 \mathrm{~h} 32^{\prime}$ & $\beta \mathrm{Uma}$ \\
2004 & $\eta \mathrm{Uma}$ & $\& 656$ & - & $22 \mathrm{~h} 27^{\prime}$ & $\eta \mathrm{Uma}$ \\
\hline \multirow{2}{*}{$22 \mathrm{nd}$} & $\beta \mathrm{Uma}$ & - & 11.9 & $19 \mathrm{~h} 49^{\prime}$ & $\beta \mathrm{Uma}$ \\
April & $\eta \mathrm{Uma}$ & 529 & - & $21 \mathrm{~h} 00^{\prime}$ & $\eta \mathrm{Uma}$ \\
2004 & $\epsilon \mathrm{Uma}$ & $\&$ & - & $22 \mathrm{~h} 22^{\prime}$ & $\eta \mathrm{Uma}$ \\
& $\alpha \mathrm{Uma}$ & 656 & 32 & $23 \mathrm{~h} 15^{\prime}$ & $\eta \mathrm{Uma}$ \\
& $\alpha \mathrm{CrB}$ & - & 14.4 & $00 \mathrm{~h} 55^{\prime}$ & $\alpha \mathrm{CrB}$ \\
\hline
\end{tabular}

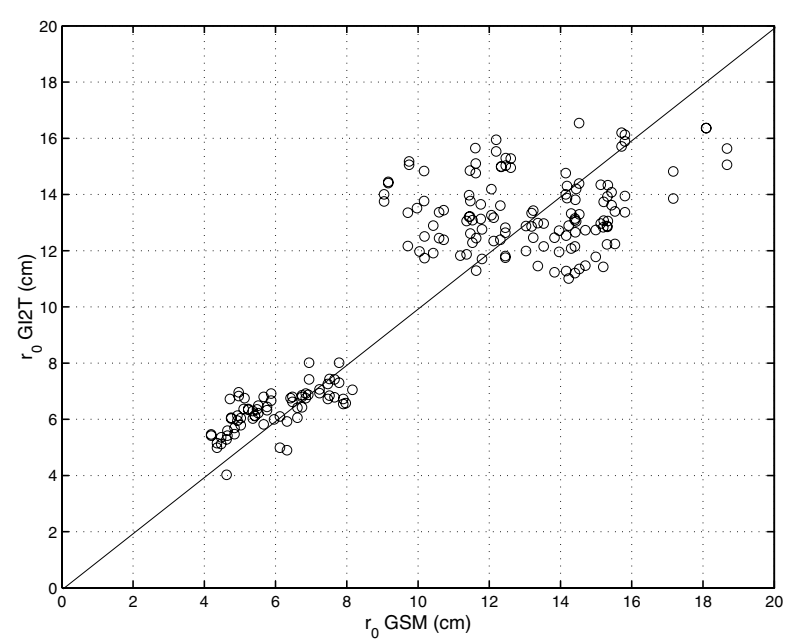

Fig. 4. Comparison of $r_{0}$ measurements obtained with the GSM and derived from the GI2T interferometer data obtained on 21st and 22nd April 2004. The correlation coefficient is equal to 0.89 .

methods at two different wavelengths. The spectral bandwidth used was $35 \mathrm{~nm}$ and the sampling frequency was 50 images/s.

We repeated simultaneous observations in order to verify the reliability of the methods under different weather and seeing conditions; the first night had a clear sky but we remarked a high level of humidity at ground level. During the second night, the sky was slightly misty and there was weak ground wind. On the third night, the sky was clear and there was no ground wind.

\subsection{Comparison of $r_{0}$ measurements}

The comparison between the seeing estimation obtained with the GI2T interferometer and those measured simultaneously with the GSM instrument is presented in Fig. 4.

Each GI2T acquisition, with a typical duration of three minutes, corresponds to about 9000 short exposure images to estimate $r_{0}$ reliably. All seeing estimates are scaled to the wavelength $\lambda=0.5 \mu \mathrm{m}$ and are given for the zenith direction.

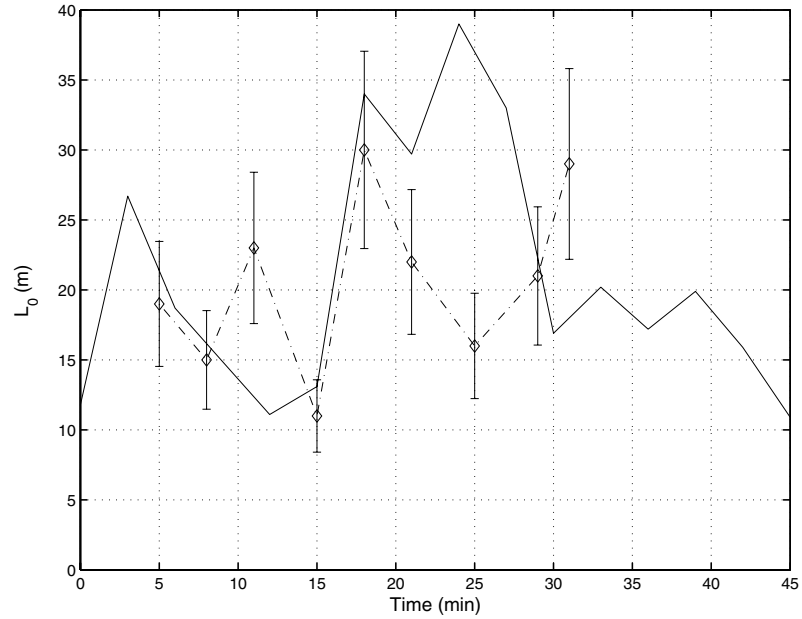

Fig. 5. Comparison of outer scale $\mathcal{L}_{0}$ measurements obtained with the GSM (solid line) and derived from the GI2T interferometer data (diamond dots) during the night of June 7th 2003. Origin is at 0h36' UT.

We found a strong correlation coefficient of 0.89 between GSM measurements and GI2T estimates.

The error on GSM $r_{0}$ measurements is $1.2 \%$ (Ziad et al. 2000). The error on the GI2T $r_{0}$ estimates is greater; this is due to the propagation inside the horizontal arms and can be due to the long exposure time of the camera (Sect. 3.3.2). The horizontal propagation leads to an underestimation of $r_{0}$ and the exposure time leads to an overestimation of it. Excellent agreement is found when the first effect compensates for the second one.

Some good seeing conditions were present on the night of April 22nd that is unusual for this observatory. Statistics for this site show that the probability of having a seeing larger than $10 \mathrm{~cm}$ is $20 \%$ (Bonneau 1997).

\subsection{Comparison of $\mathcal{L}_{0}$ measurements}

Substituting standard deviations of the OPD obtained from GI2T data into Eq. (3) with GSM $r_{0}$ measurements leads to estimations of the outer scale presented in Fig. 5 for the night of June 07th 2003 and in Fig. 6 for the night of April 22nd 2004. Each GI2T estimation presented in Fig. 6 is a mean value of results obtained with the red and blue channels. These results are in very close agreement, $\mathcal{L}_{0}$ being achromatic. These estimates are compared with $\mathcal{L}_{0}$ measured by GSM. A good agreement is seen despite the difference in the two instrument scales. One can choose to use GI2T $r_{0}$ estimates, as mentioned above, for a completely independent interferometric seeing monitor. The relative uncertainty on $\mathcal{L}_{0}$ estimates deduced from Sect. 3.3.1 and GSM error on $r_{0}$ measurement is about $\pm 25 \%$. If $r_{0}$ deduced from GI2T data is used, this uncertainty is greater and it increases with the baselength (Sect. 3.3.2).

\section{Discussion and perspectives}

The results presented here show the possibility of measuring the outer scale and seeing from long-baseline interferometric data. We have shown that the GI2T, a dispersed fringe mode 


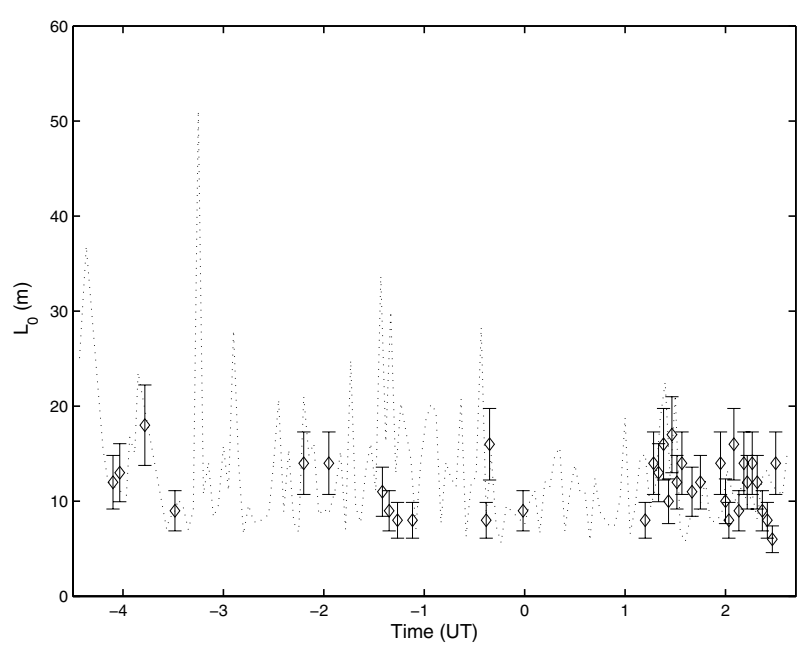

Fig. 6. Comparison of outer scale $\mathcal{L}_{0}$ measurements obtained with the GSM (dotted line) and derived from the GI2T interferometer data during the night of 22nd April 2004: diamond markers are mean values obtained from the blue and red channel.

interferometer, in addition to being a powerful astronomical instrument, is also capable of providing reliable measurements of wavefront spatial coherence parameters. Estimated values of $r_{0}$ and $\mathcal{L}_{0}$ obtained at several baselines from GI2T data are in good agreement with GSM measurements.

These interferometric estimates could be suitable for optimizing HAR observational techniques, without the need for seeing monitors, except for an initial calibration in order to characterize instrumental contributions. It can determine, for example, the SNR imposed by the turbulence for upcoming observations, or the magnitude limit which depends upon the seeing conditions (Roddier 1988). Variations of $\mathcal{L}_{0}$ should be considered in the specifications of the finite exposure time of a fringe sensor and the finite response time of a delay line (Conan et al. 2000). These estimates also could be convenient in acquiring reliable seeing statistics for a particular site with the goal to implement new HAR instruments like adaptive optics or fringe trackers.

In contrast to outer scale estimates, OPD fluctuations are obtained without the need for an atmospheric turbulence model. The three points in Fig. 7 represent mean values of $\sigma_{\text {OPD }}$ for three baselines used sequentially on the night of 22nd June, 2004. Error bars correspond to the standard deviation of $\sigma_{\mathrm{OPD}}$ obtained for each baseline performed. As remarked by Mariotti \& di Benedetto (1984) and then by Davis et al. (1995), the departure from the Kolmogorov model is significantly pronounced for long baselines. The non-simultaneity of our measurements makes it difficult to draw a conclusion about the validity of the VK model on decametric baselines. It is noticeable however that the saturation of $\sigma_{\mathrm{OPD}}$ and hence the saturation of the phase structure function is highlighted for baselines longer than the outer scale.

As the VLTI/Amber is a dispersed fringe mode interferometer, methods used in this paper could be applied to its data. The advantage of this instrument is that some instrumental contribution to the OPD are known and that others could be evaluated by specific measurements. Inside the VLTI, fringe signals

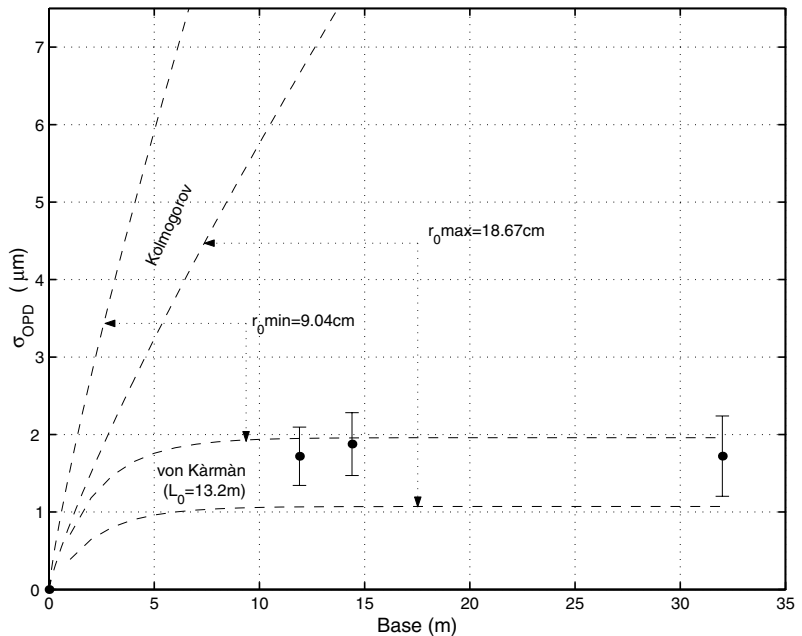

Fig. 7. Standard deviation of OPD fluctuations as a function of baseline. The three points are the mean measurements performed sequentially on the night of the 22nd June 2004 obtained with the GI2T interferometer. Dashed lines represent $\sigma_{\mathrm{OPD}}$ in cases of Kolmogorov and VK models for extreme values of $r_{0}(9.0 \mathrm{~cm}$ and $18.6 \mathrm{~cm})$ measured by GSM during OPD acquisition. Using the VK model, we represent $\sigma_{\mathrm{OPD}}$ in the case of the outer scale median value $\mathcal{L}_{0}=13.2 \mathrm{~m}$ measured by GSM during OPD acquisition.

are affected by static optical path differences and also by timevarying OPD fluctuations introduced by the motion of delay lines, by vibrations of mechanical structures and by air turbulence. It is possible to measure in real-time all of these internal contributions. With 3 baselines available simultaneously, the VLTI/Amber interferometer makes makes it possible to considerably improve our knowledge of phase fluctuations at decametric and hectometric scales and therefore makes possible the validation of atmospheric turbulence models.

\section{Appendix A: Theoretical background}

Statistics of phase fluctuations follow that of temperature and refractive index inhomogeneities. As long as the base length $B$ is included in the inertial range that is limited by the turbulence inner and outer scales, the phase structure function is governed by the Kolmogorov law which leads to the phase power spectrum,

$W_{\phi}^{\mathrm{K}}(\vec{u})=0.0229 r_{0}^{-5 / 3} u^{-11 / 3}$

where $u$ is the modulus of the spatial frequency $\vec{u}$. $r_{0}$ depends physically on the structure constant of refractive index fluctuations $C_{N}^{2}(h)$ integrated along the propagation path (Roddier 1981) as,

$r_{0}=\left[0.423\left(\frac{2 \pi}{\lambda}\right)^{2}(\cos \gamma)^{-1} \int C_{N}^{2}(h) \mathrm{d} h\right]^{-3 / 5}$.

In the framework of this model and without pupil filtering effects, the phase structure function can be rewritten as (Roddier 1981)

$D_{\phi}^{\mathrm{K}}(\vec{B})=6.88\left(B / r_{0}\right)^{5 / 3}$ 
The VK model formalizes the long-baseline saturation of the phase structure function and its phase power spectrum is given by (Borgnino et al. 1992)

$$
W_{\phi}^{\mathrm{VK}}(\vec{u})=0.0229 r_{0}^{-5 / 3}\left[u^{2}+\frac{1}{\mathcal{L}_{0}^{2}}\right]^{-11 / 6} \text {. }
$$

Using this model, Conan et al. (2000) derived asymptotic solutions for Eq. (2) in three regimes:
(a) $D \ll B \ll \mathcal{L}_{0} / \pi$ :

$$
D_{\phi}^{\mathrm{VK}}(\vec{B})=6.88\left(\frac{B}{r_{0}}\right)^{5 / 3}\left[1-1.49\left(\frac{B}{\mathcal{L}_{0}}\right)^{1 / 3}\right]
$$$$
\text { (b) } D \ll \mathcal{L}_{0} / \pi \ll B: D_{\phi}^{\mathrm{VK}}(\vec{B})=0.17\left(\frac{\mathcal{L}_{0}}{r_{0}}\right)^{5 / 3}
$$$$
\text { (c) } \mathcal{L}_{0} / \pi \ll D \ll B: D_{\phi}^{\mathrm{VK}}(\vec{B})=0.06\left(\frac{D}{r_{0}}\right)^{5 / 3}\left(\frac{D}{\mathcal{L}_{0}}\right)^{-11 / 3} \text {. }
$$

The relation between $\sigma_{\mathrm{OPD}}$ obtained with the two models may be found, as was carried out by Avila et al. (1997) in the case of the AA covariance. We take into account pupil effects in the VK model but not in the Kolmogorov model. In this latter case, the telescope diameter can be considered infinite. Substituting Eq. (A.4) into Eq. (2) and substituting $x$ for the dimensionless variable $2 \pi B u$ leads, with respect to the VK model, to

$$
\begin{array}{rl}
D_{\phi}^{\mathrm{VK}}(\vec{B})=0 & .287 r_{0}^{-5 / 3} \int_{0}^{+\infty} \frac{x}{(2 \pi B)^{2}} \\
& \times\left[\frac{x^{2}}{(2 \pi B)^{2}}+\left(\frac{1}{\mathcal{L}_{0}}\right)^{2}\right]^{-11 / 6}\left[1-J_{0}(x)\right] \\
& \times\left[\frac{2 J_{1}\left(\frac{D x}{2 B}\right)}{\frac{D x}{2 B}}\right]^{2} \mathrm{~d} x .
\end{array}
$$

Taking Eq. (3) into account leads to the expression of $\sigma_{\mathrm{OPD}}$ in the framework of the VK model as

$\sigma_{\mathrm{OPD}}^{\mathrm{VK}}(\vec{B})=\sigma_{\mathrm{OPD}}^{K}(\vec{B}) \cdot\left[H_{\phi}^{\mathrm{VK}}(B)\right]^{1 / 2}$

where $\sigma_{\mathrm{OPD}}^{\mathrm{K}}(\vec{B})$ is the OPD standard deviation for the Kolmogorov model when the pupils are assumed to be infinitely small. $H_{\phi}^{\mathrm{VK}}(B)$ is the distortion function which includes filtering effects that are due to the outer scale and to the pupil size, as

$$
\begin{aligned}
H_{\phi}^{\mathrm{VK}}(B)=0.894 & \int_{0}^{+\infty} x\left[x^{2}+\left(\frac{2 \pi B}{\mathcal{L}_{0}}\right)^{2}\right]^{-11 / 6} \\
& \times\left[1-J_{0}(x)\right]\left[\frac{2 J_{1}\left(\frac{D x}{2 B}\right)}{\frac{D x}{2 B}}\right]^{2} \mathrm{~d} x .
\end{aligned}
$$

Figure A.1 represents $\sigma_{\mathrm{OPD}}^{\mathrm{VK}}$ as a function of the dimensionless variable $B / \mathcal{L}_{0}$, given in units of $\lambda\left(B / r_{0}\right)^{5 / 6}$, for different values of the ratio $\mathrm{D} / B$. The value at the origin, i.e. when $\mathcal{L}_{0} / B$ tends to infinity, is $\sigma_{\mathrm{OPD}}^{\mathrm{VK}}(0)=0.417$ : it corresponds to the case $H_{\phi}^{\mathrm{VK}}(B)=1$; at this point, the phase structure function is equivalent in both $\mathrm{VK}$ and Kolmogorov models. $\sigma_{\mathrm{OPD}}^{\mathrm{VK}}$ in units of $\lambda\left(B / r_{0}\right)^{5 / 6}$ decreases as $B / \mathcal{L}_{0}$ increases but $\sigma_{\mathrm{OPD}}^{\mathrm{VR}}$ in units of $\lambda$ increases with $B$. The median values of $\mathcal{L}_{0}=22 \mathrm{~m}$ and $r_{0}=11.5 \mathrm{~cm}$ (at $\lambda=0.5 \mu \mathrm{m}$ ) measured at Paranal (Martin et al. 2000) give a typical value of $\sigma_{\mathrm{OPD}}$ equal to $4 \lambda$ for UT's separation of $47 \mathrm{~m}$. Considering $\mathcal{L}_{0}=25 \mathrm{~m}, r_{0}=10 \mathrm{~cm}$ and a $12 \mathrm{~m} \mathrm{GI} 2 \mathrm{~T}$ baseline leads to $\sigma_{\mathrm{OPD}}=6 \lambda$.

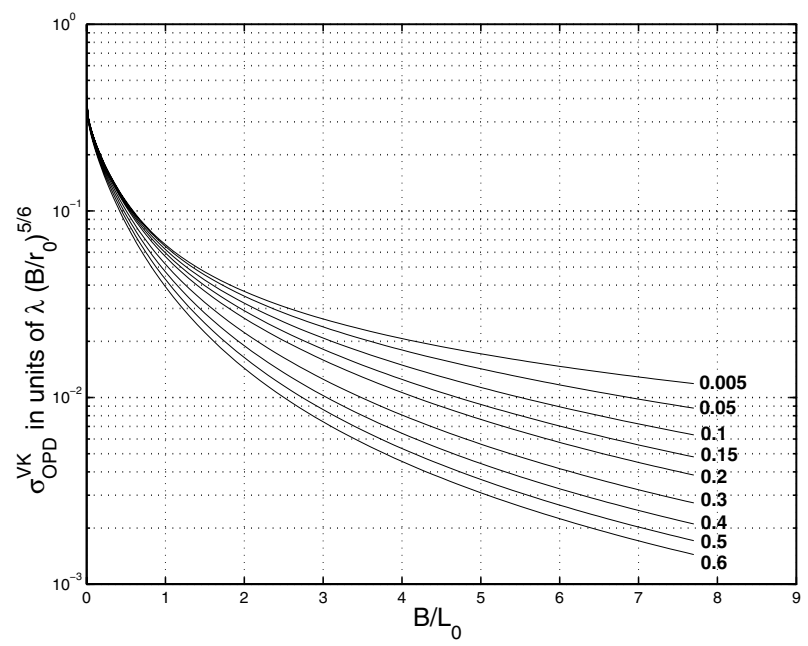

Fig. A.1. Standard deviation of the OPD, in units of $\lambda\left(B / r_{0}\right)^{5 / 6}$, as a function of the dimensionless parameter $B / \mathcal{L}_{0}$ for different values of the ratio $\mathrm{D} / B$, obtained from numerical integration of Eq. (A.7).

Acknowledgements. The authors gratefully acknowledge the referee for relevant comments and constructive suggestions. We also thank C. Aime, H. Lantéri and F. Vakili for helpful comments. We are grateful to the technical team of the GI2T interferometer, especially J. L. Chevassut for his part in the successful observations.

\section{References}

Aime, C. 2001, Eur. J. Phys., 22, 169

Aime, C., Kadiri, S., Ricort, G., Roddier, C., \& Vernin, J. 1979, Opt. Acta, 26, 575

Avila, R., Ziad, A., Borgnino, J., et al. 1997, J. Opt. Soc. Am. A, 11, 3070

Berio, P., Mourard, D., Vakili, F., Borgnino, J., \& Ziad, A. 1997, J. Opt. Soc. Am. A, 14, 114

Berio, P., Vakili, F., Mourard, D., \& Bonneau, D. 1998, A\&AS, 129, 609

Bester, M., Danchi, W., Degiacomi, C., Greenhill, L., \& Townes, C. 1992, ApJ, 392, 357

Bonneau, D. 1997, J. Astron. Fr., 52, 35

Borgnino, J. 2004, EAS Public. Ser., 12, 103

Borgnino, J., Aime, C., Martin, F., Petrov, R. G., \& Ricort, G. 1989, J. Opt. Soc. Am. A, 6, 244

Borgnino, J., Martin, F., \& Ziad, A. 1992, Opt. Com., 91, 267

Buscher, D., Armstrong, J., Hummel, C., et al. 1995, Appl. Opt., 34, 1081

Colavita, M. M., Shao, M., \& Staelin, D. H. 1987, Appl. Opt., 26, 4106

Conan, R., Ziad, A., Borgnino, J., Martin, F., \& Tokovinin, A. A. 2000, in Proc. SPIE, 4006, 963

Davis, J., Lawson, P., Booth, A., Tango, W., \& Thorvaldson, E. 1995, MNRAS, 273, L53

Frieden, B. R. 1983, Probability, Statiscal optics, and data testing (Springer-Verlag)

Fusco, T., Rousset, G., Rabaud, D., et al. 2004, J. Opt. A: Pure Appl. Opt., 6, 585

Haniff, C. A., Baldwin, J. E., Warner, P. J., \& Scott, T. R. 1994, Proc. SPIE, 2200, 407

Koechlin, L., Lawson, P. R., Mourard, D., et al. 1996, Appl. Opt., 35, 3002 
Linfield, R. P., Colavita, M. M., \& Lane, B. F. 2001, ApJ, 554, 505

Mariotti, J. M., \& di Benedetto, G. P. 1984, in IAU Coll., 79, 257

Martin, F., Conan, R., Tokovinin, A., et al. 2000, A\&AS, 144, 39

Martin, F., Tokovinin, A., Agabi, A., Borgnino, J., \& Ziad, A. 1994, A\&AS, 108, 173

Mourard, D., Tallon-Bosc, I., Rigal, F., et al. 1994, A\&A, 288, 675

Mourard, D., Thureau, N., Abe, L., et al. 2001, C.R. Acad. Sci. Paris, 2,35

Nightingale, N., \& Buscher, D. 1991, MNRAS, 251, 155

Parks, T., \& Burrus, C. 1987, Digital Filter Design (New York: John Wiley and Sons)
Roddier, C., \& Roddier, F. 1975, J. Opt. Soc. Am., 65, 664

Roddier, F. 1981, in Progress in Optics, Vol. XIX

Roddier, F. 1988, Phys. Rep., 170, 99

Roddier, F., \& Lena, P. 1984, J. Optics (Paris), 15, 171

Sarazin, M., \& Roddier, F. 1990, A\&A, 227, 294

Schöck, M., Le Mignant, D., Chanan, G.-A., Wizinowich, P.-L., \& van Dam, M.-A. 2003, Appl. Opt., 42, 3705

Ziad, A., Conan, R., Tokovinin, A., Martin, F., \& Borgnino, J. 2000, Appl. Opt., 39, 5415

Ziad, A., Schöck, M., Chanan, G. A., et al. 2004, Appl. Opt., 43, 2316 